\title{
SUPERMASSIVE BLACK HOLE BINARIES AT HIGH ENERGIES*
}

\author{
GUSTAVO E. ROMERO $^{\dagger, \ddagger, \S}$, DANIELA PÉREZ ${ }^{\dagger, \uparrow}$ and GABRIELA S. VILA ${ }^{\dagger, \|}$ \\ $\dagger$ Instituto Argentino de Radioastronomía, \\ C.C. 5, (1894) Villa Elisa, Buenos Aires, Argentina \\ ${ }^{\ddagger}$ Facultad de Cs. Astronómicas y Geofísicas, Universidad Nacional de La Plata, \\ Paseo del Bosque S/N, (1900) La Plata, Buenos Aires, Argentina \\ §romero@iar-conicet.gov.ar \\ Idanielaperez@iar-conicet.gov.ar \\ " gvila@iar-conicet.gov.ar
}

Received 1 November 2013

Revised 18 November 2013

Published 21 March 2014

\begin{abstract}
An accretion disk around a supermassive black hole may be strongly perturbed by the presence of a secondary black hole. Recent simulations have shown that, under certain conditions, the tidal torques exerted by the secondary black hole may open an annular gap in the disk. In this regime, matter "overflows" across the secondary's orbit to accrete onto the primary and may feed a pair of relativistic jets.

In this work we study the radiative properties of a binary system of supermassive black holes, assuming that a relativistic jet is launched from the primary and the migration of the secondary across the disk proceeds in the "overflowing" regime. The modified radiative spectrum of the disk is calculated accounting for strong gravitational effects in the innermost region. The jet emits non-thermal radiation all along the electromagnetic spectrum by interaction of locally accelerated electrons with the jet's magnetic field and internal and external radiation. In particular, we investigate whether the interaction of the relativistic electrons with the photons emitted by the accretion disk induces any signature in the spectral energy distribution of the jet that may reveal the presence of the secondary black hole.
\end{abstract}

Keywords: Supermassive black hole binaries; astrophysical jets; radiation mechanisms: non-thermal.

\section{Introduction}

The formation of a binary system of supermassive black holes is the likely outcome of the merger of two galaxies. ${ }^{1,2}$ During the late stages of their evolution, supermassive black hole binaries (SMBHBs) are expected to be strong sources of gravitational

* This is an Open Access article published by World Scientific Publishing Company. It is distributed under the terms of the Creative Commons Attribution 3.0 (CC-BY) License. Further distribution of this work is permitted, provided the original work is properly cited. 
waves at low and very low frequencies, and thus are suitable targets for space interferometers and pulsar timing arrays. ${ }^{3}$

Currently there are less than 20 SMBHBs identified but there is a large number of candidates. The known SMBHBs have orbital separations from about $10 \mathrm{kpc}$ down to $\sim 7$ pc. Those with the largest orbital separation may be directly resolved in $\mathrm{X}$ rays as two distinct active nuclei in the same galaxy. Other usual identification criteria include periodicity in the lightcurve (as in the case of the very studied BL Lac Object OJ 287, see e.g. Ref. 4) and blue or red-shifted broad lines. Notice, however, that binarity is not the only possible explanation for these features and hence many identifications are not conclusive.

The process of migration of the secondary (the less massive) black hole towards the primary (the more massive) resembles planetary migration in many aspects. For instance, depending on the masses of the black holes and their separation, the secondary may clear a gap (a region of very low mass density) in the disk, see e.g. Refs. 5, 6 and 7. Recent numerical simulations of binary supermassive black holes where the secondary is embedded in the accretion of the primary are presented in Refs. 8 and 9. The authors compute the coupled evolution of the structure of the disk and the orbital separation under the action of viscous and tidal torques and gravitational wave emission. They find that for certain combinations of the values of the mass ratio and the orbital separation, the system relaxes to a quasi-steady state in which an annular gap develops in the disk about the orbit of the secondary. The inner part of the disk, however, in not empty, since matter is able to flow across the secondary's orbit. In this so-called "overflowing" regime, the region of the disk close to the gap is significantly perturbed by the presence of the secondary. In particular, there is an accumulation of matter outside the secondary's orbit that causes the disk to become hot and thick. The radiative spectrum of such a disk is different from that of a standard geometrically thin and optically thick disk, as shown in Refs. 7, 9 and 10 among others.

A fraction of the matter that fills the inner disk is accreted onto the primary and may feed a pair of relativistic jets. One of the most efficient mechanisms of high-energy non-thermal emission from relativistic jets is inverse Compton (IC) scattering of photons off relativistic electrons. The target radiation fields may be internal (synchrotron photons, in which case the process is dubbed synchrotron self-Compton, SSC) or external (external Compton, EC). An important source of low-energy photons external to the jet is the accretion disk. These photons may also absorb the high-energy radiation emitted in the jet to create pairs. The characteristics of the IC spectrum and the opacity to gamma-ray propagation depend on the energy distribution of target photons, so any noticeable feature in the radiative spectrum of the disk is expected to have a correlation in the high-energy spectrum of the jets.

Here we consider a binary system of supermassive black holes in the "overflowing" regime where the primary launches a relativistic jet. We calculate the modified radiative spectrum of the disk applying the results of the simulations in Refs. 8, 
9 to characterize its structure. We then compute the non-thermal spectral energy distribution (SED) of the jet including the external IC scattering of disk photons and its modification by absorption. Finally, we assess if the high-energy part of the SED displays any features that could disclose the presence of the secondary.

\section{Basics of the Model and Results}

We consider a supermassive black hole binary where the orbit and the accretion disk are coplanar as sketched in Figure 1. The primary is a Kerr black hole of mass $M$ and spin parameter $a=0.99$. The mass of the secondary is $m_{\mathrm{s}}$; it is located at a constant distance $r_{\mathrm{s}}$ from the primary (we fix $r=0$ at the position of $M$ ). We assume that the secondary has opened a steady annular gap in the disk around $r=r_{\mathrm{s}}$. The half width of the gap is approximately equal to the Hill radius $r_{\mathrm{H}}$ of the secondary, given by

$$
\Delta r_{\text {gap }} \approx r_{\mathrm{H}}=r_{\mathrm{s}}\left(\frac{q}{3}\right)^{1 / 3}
$$

where $q \equiv m_{\mathrm{s}} / M \ll 1$ is the mass ratio.

The results of the simulations by Refs. 8,9 show that the accretion disk may be divided into five regions with different characteristics. The influence of the secondary is negligible in the inner far and outer far regions; here the disk is well described as a standard Shakura-Sunyaev disk. ${ }^{11}$ The inner far region, however, is under the strong gravitational field of the primary, so we apply the relativistic version of the Shakura-Sunyaev model ${ }^{12}$ to model this zone of the disk. In the near interior, near exterior and middle regions the disk is perturbed by the presence of the secondary. Matter piles up in the near exterior (and also the middle) region, that heats and inflates. For the mass density and surface temperature of the disk as a function of the radius in these zones we adopt the parametrizations given in Refs. 8, 9, that are fits to the results of the simulations.

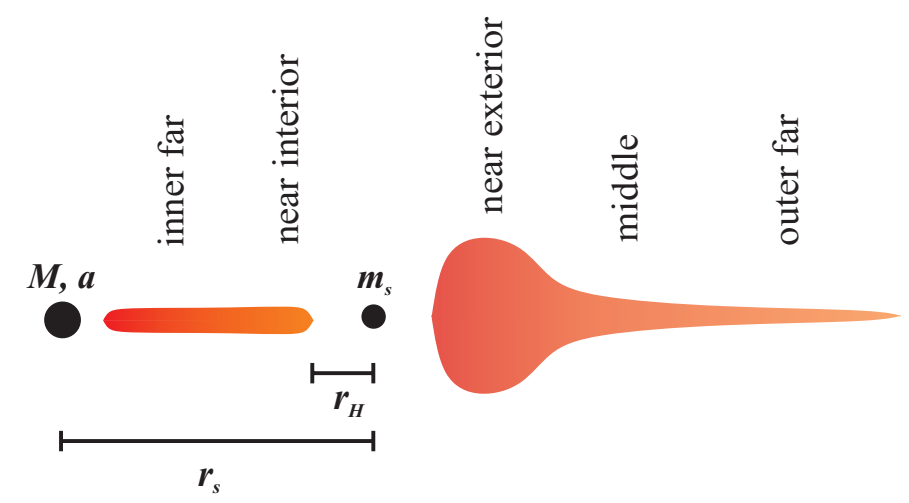

Fig. 1. A SMBHB where the secondary has cleared an annular gap in the accretion disk. Five regions with different characteristics may be identified in the disk. 
We estimate the transition radius between the inner far and the near interior regions equating the mass density on both sides, and apply the formula given in Ref. 8 to calculate the transition radius between the near exterior and the middle regions. Notice that the simulations of Refs. 8, 9 are not valid inside the gap, so the region $r_{\mathrm{s}}-r_{\mathrm{H}} \leq r \leq r_{\mathrm{s}}+r_{\mathrm{H}}$ is excluded from our calculations.

We calculate the temperature profile of the disk and the SEDs of the disk and the jet for several values of $M, q$ and $r_{\mathrm{s}}$ for which the model in Refs. 8, 9 predicts that the system is in the "overflowing" regime. Figure 2 shows the temperature profile as a function of radius and the SED of the disk for a model with $M=10^{7} M_{\odot}, q=5.5 \times 10^{-3}, r_{\mathrm{s}}=18000 r_{\mathrm{g}}$, and mass accretion rate onto the primary $\dot{M}=0.1 \dot{M}_{\text {Edd }}$. Here $r_{\mathrm{g}}$ and $\dot{M}_{\text {Edd }}$ are the gravitational radius and the Eddington accretion rate of the primary black hole, respectively. A peak is clearly seen in the temperature profile at the position of the near exterior region. This causes the increase in the luminosity of the disk at low energies compared to a relativistic standard disk.

To characterize the jet we apply the model developed in Refs. 13, 14 and 15 . The jets are two conical outflows launched perpendicularly to the accretion disk and oriented at an angle $\theta_{\text {jet }}$ with the line of sight of the observer. The bulk Lorentz factor $\Gamma_{\text {jet }}$ of the outflows is assumed to be constant. The total power carried by each jet is $L_{\text {jet }}=0.05 \dot{M} c^{2}$. In some region of the jet and by some mechanism we do not specify here, a $10 \%$ of the jet power is transferred to particles that get accelerated up to relativistic energies. These particles are injected isotropically in the co-moving reference frame and follow a power-law distribution in energy. For the present applications we consider only electrons, although jets containing relativistic protons have been considered before as part of the same model in Refs. 13-15 and others. Once injected in the jet, the relativistic electrons lose energy by radiation and adiabatic loses. We calculate the steady-state distribution of electrons as a
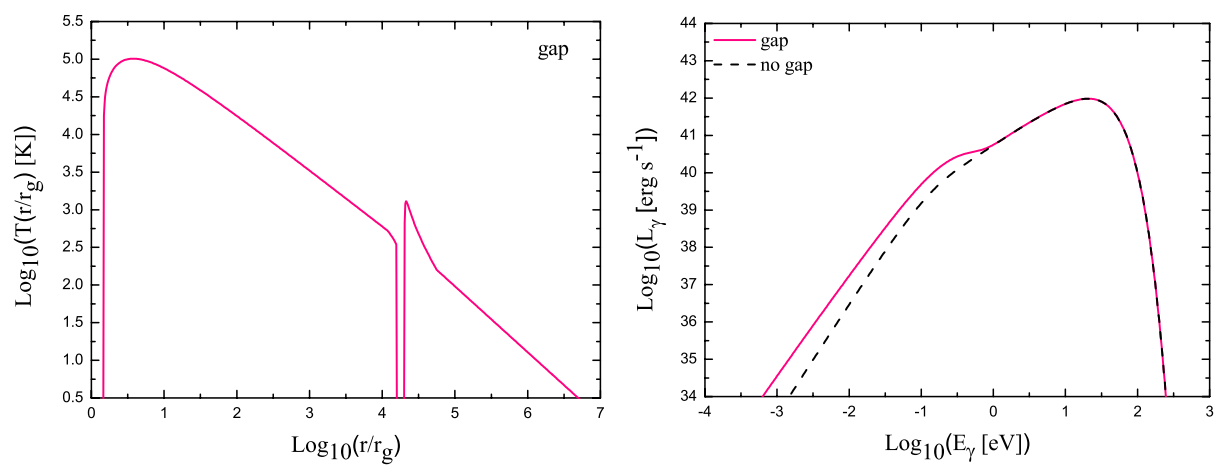

Fig. 2. Left. Surface temperature of the disk as a function of radius in units of the gravitational radius of the primary. Right. Solid line: radiative spectrum of the disk (calculated assuming it radiates as a black body at the local surface temperature) for the same set of parameters. Dashed line: spectrum predicted by the relativistic standard disk model of Page \& Thorne (1974). 

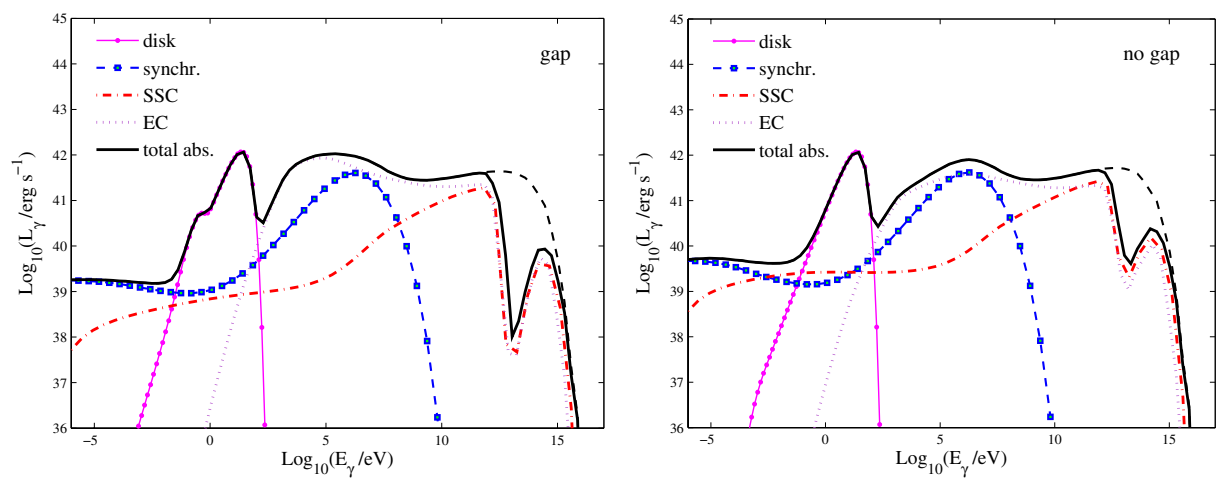

Fig. 3. Left. Broadband non-thermal SED of the jet for the case of an accretion disk with a gap. The blackbody emission of the disk is also plotted. The black curve is the total luminosity before (dashed line) and after (solid line) correcting for absorption. Right. The same but for a disk without a gap.

function of energy and distance to the primary in the co-moving frame solving a transport equation that accounts for injection, cooling and convection.

We calculate the radiative spectrum from the interaction of the relativistic electrons with the internal magnetic field of the jet and the external (disk) and internal (synchrotron) radiation fields. In computing the EC emission we took into account the anisotropy of the disk photon field as seen from the jet, see e.g. Ref. 16. The high-energy photons that escape the jet may be absorbed by photons from the disk to create electron-positron pairs. To correct the jet SED for absorption we adopt an attenuation parameter $\exp \left(-\tau_{\gamma \gamma}\right)$, where $\tau_{\gamma \gamma}\left(E_{\gamma}\right)$ is the optical depth for pair production. ${ }^{17}$ This is a first order correction to the SED; the triggering of an electromagnetic cascade remains to be investigated.

In Figure 3 we show the spectral energy distribution of the jet in a model with $\Gamma_{\text {jet }}=20, \theta_{\text {jet }}=5^{\circ}$, and a magnetic field $B \sim 2 \mathrm{G}$ in the acceleration region. For comparison the SED obtained for the same parameters but for a disk without a gap is also shown. We find basically two features that distinguish them. One is that the low energy part of the EC spectrum is slightly more luminous in the model with a disk with a gap. The second difference is that the absorption well at $\sim \mathrm{TeV}$ energies is deeper in the model with gap. Both effects are the result of the excess of target photons for scattering and absorption emitted in the near exterior region.

\section{Discussion and Perspectives}

The difference in the low-energy cutoff of the EC emission between the models with and without a gap in the disk, at least in the cases we present here, is rather weak to be unambiguously recognized as a signature of the presence of a secondary black hole. According to our results, the characteristics of the SED at very high energies (together with the features expected in the emission of the accretion disk) could provide a good criterion to support the identification of SMBHB candidates. In this 
energy range, future observations with Cherenkov arrays will be the appropriate tool to investigate the effects predicted here.

Other regions of the parameter space allowed by the "overflowing" regime remain to be explored. We expect the effects of the existence of the gap to become more pronounced for larger values of $M$. Furthermore, the presence of the secondary may induce precession in the jet. The EC emission and the absorption effects for a tilted jet could differ from the results presented here. These questions will be addressed in a forthcoming elaboration of this work.

\section{Acknowledgments}

G.S.V. wishes to thank the High-Energy Astrophysics Theory Group of the MaxPlanck-Institut für Kernphysik for their hospitality during her stay and the preparation of this work. This research was supported by grants PIP 0078/2010 and PICT 2012-00878 Préstamo BID.

\section{References}

1. S. Komossa, Mem. S. A. It. 77, 733 (2006).

2. M. Dotti, A. Sesana, R. Decarli, Adv. Ast. vol. 2012, id. 940568 (2012).

3. A. Sesana, Gravitational wave emission from binary supermassive black holes, to appear in Classical and Quantum Gravity, arXiv:1307.4086.

4. A. Sillanpää, S. Haarala, M. J. Valtonen, B. Sundelius, G. G. Byrd, ApJ 325, 628 (1988).

5. S. Syer, C. J. Clarke, MNRAS 277, 758 (1995).

6. J. Goodman, J. C. Tan, ApJ 608, 108 (2004).

7. Y. T. Liu, S. L. Shapiro, PRD 82, 123011 (2010).

8. B. Kocsis, Z. Haiman, A. Loeb, MNRAS 427, 2660 (2012a).

9. B. Kocsis, Z. Haiman, A. Loeb, MNRAS 427, 2680 (2012b).

10. K. Gültekin, J. M. Millë, ApJ 761, 90 (2012).

11. N. I. Shakura, R. A. Sunyaev, $A \& A$ 24, 337 (1973).

12. D. N. Page, K. S. Thorne, ApJ 191, 499 (1974).

13. G. E. Romero, G. S. Vila, $A \mathscr{E} A$ 485, 623 (2008).

14. M. M. Reynoso, M. C. Medina, G. E. Romero, $A \mathscr{E} A$ 531, id.A30 (2011).

15. G. S. Vila, G. E. Romero, N. A. Casco, $A \& B A$ 538, id.A97 (2012).

16. C. D. Dermer, R. Schlickeiser, ApJ 416, 458 (1993).

17. P. A. Becker, M. Kafatos, ApJ 453, 83 (1995). 